\title{
Therapeutic Opportunities for Hepcidin in Acute Care Medicine
}

\author{
Lakhmir S. Chawla, MD ${ }^{a, b, *}$, Blaire Beers-Mulroy, MS ${ }^{b}$, \\ George F. Tidmarsh, MD, PhD ${ }^{\mathrm{b}, \mathrm{c}}$
}

\section{KEYWORDS}

- Hepcidin • Catalytic free iron • Macrophage polarization • Siderotherapy

- Iron homeostasis • Infections • Ferroportin • Transferrin saturation

\section{KEY POINTS}

- Acute injury mechanisms increase catalytic free iron.

- The natural inflammatory response stimulates hepcidin production to induce serum hypoferremia to reduce the pathologic effects of increased catalytic free iron.

- Exogenous hepcidin may provide a more rapid and pronounced therapeutic benefit in the prevention and treatment of iron-induced injury during acute disease.

\section{INTRODUCTION}

Iron is a critical nutrient that is required for basic metabolic processes in cells. However, iron is a reactive transition metal and its availability in the body is carefully regulated because excess iron can increase the production of oxygen free radicals, which can cause cellular damage. ${ }^{1}$ As such, a tight control of iron availability, concentration, and tissue distribution is essential. Hepcidin is the principal regulator of iron in the body. ${ }^{2-4}$ The mature bioactive form of hepcidin is a 25 amino acid peptide that derives from the 84 amino acid precursor preprohepcidin, which undergoes two enzymatic cleavages. Ferroportin is a transmembrane protein that exports iron from inside to the outside of a cell. This channel is the primary mechanism by which iron is exported out of cells, and the regulation of ferroportin allows control of iron in cells, the plasma, extracellular space, and absorption of iron from the gut. Hepcidin exerts

Disclosure Statement: L.S. Chawla, B. Beers-Mulroy, and G.F. Tidmarsh are employees of La Jolla Pharmaceutical.

${ }^{a}$ Department of Medicine, Veterans Affairs Medical Center, La Jolla, CA 92161, USA; ${ }^{b}$ La Jolla Pharmaceutical Company, 4550 Towne Centre Court, San Diego, CA 92121, USA; ' Department of Pediatrics, Stanford University School of Medicine, Palo Alto, CA 94305, USA

* Corresponding author. San Diego Veterans Hospital, 3350 La Jolla Village Drive, San Diego, CA 92161.

E-mail address: minkchawla@gmail.com 
its control over iron by its interaction with ferroportin. Hepcidin attaches to ferroportin, which causes the internalization and degradation of ferroportin, thereby blocking the export of iron from reticuloendothelial macrophages, hepatocytes, immune cells, and duodenal enterocytes. ${ }^{4}$ Therefore, administration of hepcidin has the therapeutic potential to be used for the treatment of illnesses associated with acute or chronic iron excess.

\section{HISTORY OF DISCOVERY}

In 2000, two independent research groups identified a novel defensin-like disulfidebonded 25 amino acid peptide with antimicrobial activity using a mass spectrometric assay in human ultrafiltrate and urine.$^{5,6}$ Krause and colleagues ${ }^{5}$ named the novel peptide liver-expressed antimicrobial peptide (LEAP-1) after determining the expression pattern is highest in the liver and identifying its dose-dependent antimicrobial effect against bacteria and yeast. While purifying cysteine-rich antimicrobial peptides from human urine, Park and colleagues ${ }^{6}$ identified the same peptide and isolated two other amino-terminal shortened forms. The peptide was named hepcidin and determined to contain eight disulfide bonded cysteines forming a structure of $\beta$-turns, loops, and distorted $\beta$-sheets. ${ }^{6}$

The first connection between hepcidin and iron was made with the identification of dose-dependent hepcidin mRNA transcript induction by suppressive subtractive hybridization between iron-overloaded and control mice. ${ }^{2}$ This link was strengthened when Nicolas and colleagues ${ }^{3}$ demonstrated that in the absence of hepcidin, iron accumulated primarily in the liver and pancreas; moderately to the heart; that serum iron increased; and splenic iron stores depleted. These studies suggested that hepcidin could be the regulatory signal maintaining iron balance. Taken together with the identification of two mutations (93delG and $166 \mathrm{C} \rightarrow \mathrm{T}$ ) in hepcidin on $19 q 13$ in patients with juvenile hemochromatosis, Roetto and colleagues, ${ }^{7}$ linked hepcidin inactivation and iron dysregulation in humans.

The mechanism by which hepcidin mediates iron homeostasis was identified in 2004 when Nemeth and colleagues ${ }^{4}$ added hepcidin to fluorescently labeled ferroportin and observed the altered distribution of ferroportin from the cell membrane to intracellular lysosomes. This internalization and degradation of ferroportin blocked the iron exportation from duodenal enterocytes, macrophages, and hepatocytes, providing the basis for regulating the concentration and tissue distribution of iron.

\section{HEPCIDIN MECHANISM OF ACTION}

The mechanism by which hepcidin regulates iron homeostasis is well understood. ${ }^{8-10}$ Hepcidin binds to ferroportin, induces covalent modification, and causes its internalization and degradation in lysosomes, which blocks the export of iron from reticuloendothelial macrophages, hepatocytes, and duodenal enterocytes. ${ }^{4}$ Hepcidin reduces the concentration of ferroportin molecules on the cell surface thereby inhibiting the entry of iron into plasma. Limiting iron efflux from cells coupled with the ongoing iron uptake of erythropoiesis by the bone marrow results in decreased serum iron and a decreased transferrin (TF) saturation (Fig. 1). Prolonged high levels of hepcidin can result in insufficient serum iron for erythropoiesis and lead to iron-restricted anemias. ${ }^{11,12}$

Synthesis of hepcidin from hepatocytes is stimulated by elevated serum iron and by increased inflammation, and downregulated by anemia and hypoxia. ${ }^{10,13}$ Regulation of hepcidin by iron occurs through TF-bound signaling extracellularly and by hepatic iron stores. In high extracellular TF-bound iron states, human hemochromatosis protein preferentially binds TF receptor-2, which activates the BMP/SMAD pathway, 

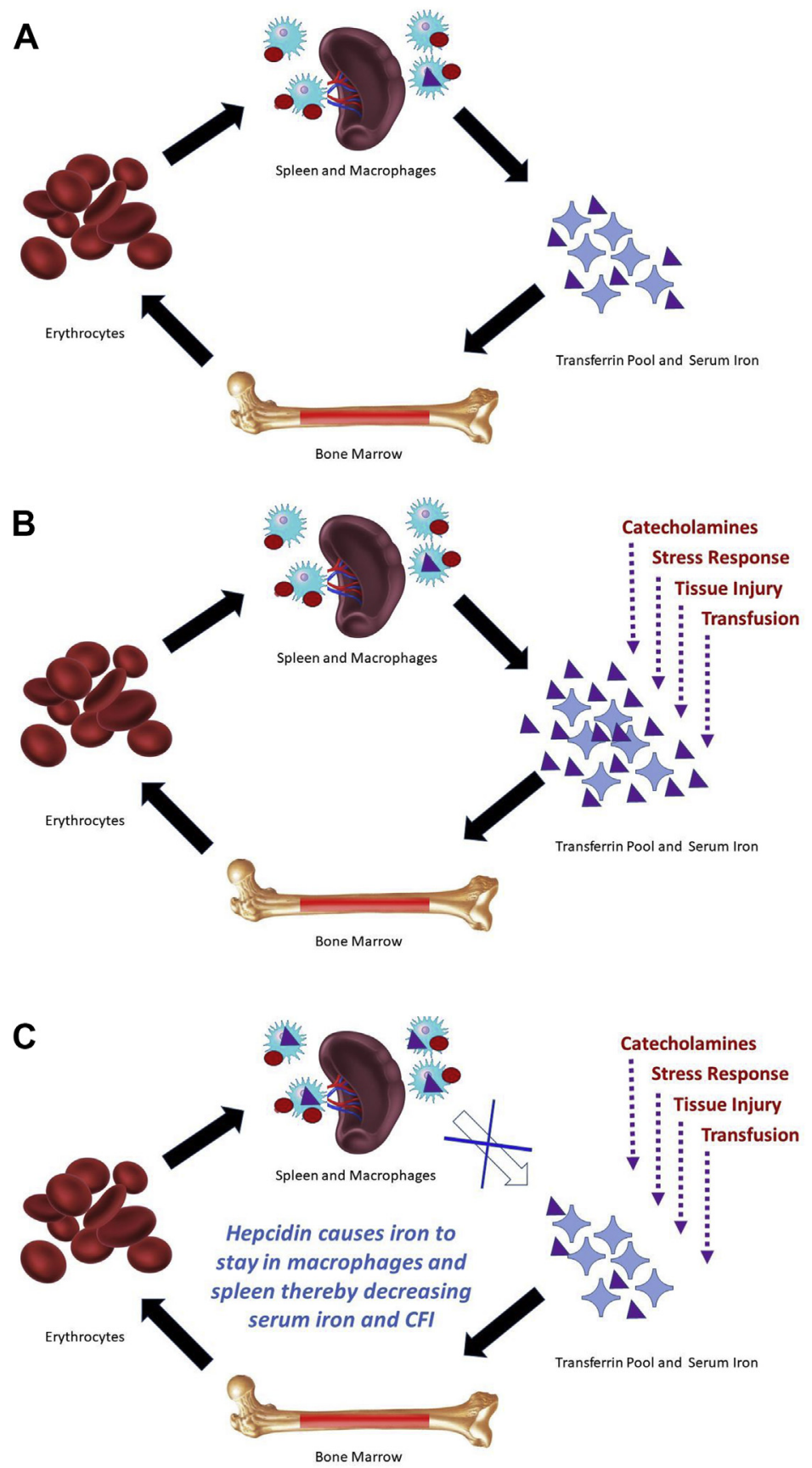

Fig. 1. Iron dynamic equilibrium in the normal and acute injury state. $(A)$ In the normal state, serum iron is bound and held under tight control by transferrin. Most serum iron is transported to the bone marrow where it is used for heme synthesis. Splenic macrophages recycle senescent erythrocytes and store iron or release it back into circulation via ferroportin. $(B)$ During stress response, catecholamines, tissue injury, and transfusion all contribute to the acute stress iron state marked by a dramatic increase in catalytic free iron. (C) Hepcidin degrades ferroportin and effectively blocks the efflux of iron from splenic macrophages, reducing the transferrin saturation. CFI, catalytic free iron. 
ultimately inducing hepcidin expression. ${ }^{14,15}$ High hepatocellular iron states increase BMP6 expression, which acts as an autocrine factor, binding the hemojuvelin and bone morphogenic protein receptor complex on hepatocytes thereby activating the SMAD pathway and inducing hepcidin expression. ${ }^{15-18}$ In iron-depleted states, transmembrane protease serine 6 degrades hemojuvelin, inhibiting hepcidin transcription. ${ }^{19,20}$

Hepcidin is also regulated by inflammation with the increase in hepcidin expression predominantly stimulated by interleukin (IL)-6. Under noninflammatory conditions, serum iron levels largely determine the serum hepcidin concentration; however, the stimulation of IL-6 to increase hepcidin supersedes the signaling from the serum iron levels. Thus, inflamed patients can often have elevated serum hepcidin levels and a low serum iron concentration. IL-6 acts as an autocrine or paracrine factor binding the IL-6 receptor on hepatocytes or reticuloendothelial macrophages, which activates the janus kinase and STAT3 signaling pathway, inducing hepcidin expression, and reducing serum iron. ${ }^{21}$ Activation of the BMP/SMAD pathway also influences hepcidin production during the inflammatory response. ${ }^{18}$ Studies have shown that an infusion of IL- 6 in humans caused an increase in hepcidin associated with an average $34 \%$ decrease in serum iron and $33 \%$ decrease in TF saturation within a few hours following infusion. ${ }^{22}$ When levels of inflammatory cytokines remain elevated in cases of chronic inflammation, hepcidin expression remains elevated, depleting serum iron required for erythropoiesis and resulting in anemia of chronic disease. Conversely, low serum iron, anemia, and hypoxia all decrease hepcidin production, resulting in a greater export of iron through ferroportin into plasma (Box 1). ${ }^{10,13}$

\section{CATALYTIC FREE IRON IN ACUTE ILLNESS}

Iron is a critical nutrient and under normal physiologic conditions catalytic free iron (CFI) is undetectable and plasma iron is tightly bound to TF. CFI, which can be thought of as unbound iron, is a promiscuous molecule that functions as a "bad-actor." When $\mathrm{CFI}$ is in its ferrous state $\left(\mathrm{Fe}^{2+}\right), \mathrm{CFI}$ can initiate the Fenton reactions or when iron is unbound in its ferric state $\left(\mathrm{Fe}^{3+}\right) \mathrm{CFI}$ can initiate Haber-Weiss reactions (Fig. 2). These reactions generate oxygen free radicals, such as hydroxyl radical $\left(\mathrm{OH}^{-}\right)$, peroxynitrite $\left(\mathrm{ONOO}^{-}\right)$, superoxide free radical anion $\left(\mathrm{O}_{2}^{-}\right)$, and hydrogen peroxide $\left(\mathrm{H}_{2} \mathrm{O}_{2}\right) .{ }^{23,24}$ These oxygen free radicals are often referred to as reactive oxygen species (ROS) and can damage important macromolecules. ROS have been shown to cause lipid peroxidation, endothelial injury, protein oxidation, mitochondrial injury, erythrocyte damage, and DNA damage (Fig. 3). ${ }^{23}$ As such, human physiologic systems

\section{Box 1 \\ Mechanisms of hepcidin therapy in acute illness}

1. Decrease catalytic free iron

a. Decreases reactive oxygen species

b. Decreases iron availability to pathogens

2. Increase serum ferritin

3. Increase intracellular H-type ferritin

4. Induce protective acute phase reactant proteins

5. Alter immune cells favorably

a. Decreases inflammatory response

b. Adjusts polarization of macrophages to $M 2$ phenotype 


$$
\begin{array}{ll}
\mathrm{Fe}^{3+}+\mathrm{O}_{2}^{-} & \longrightarrow \mathrm{Fe}^{2+}+\mathrm{O}_{2} \\
\mathrm{Fe}^{2+}+\mathrm{H}_{2} \mathrm{O}_{2} \longrightarrow \mathrm{Fe}^{3+}+\mathrm{HO}^{\circ}+\mathrm{OH}^{-} \text {(Fenton reaction) } \\
\mathrm{O}_{2}+\mathrm{H}_{2} \mathrm{O}_{2} \longmapsto \mathrm{O}_{2}+\mathrm{HO}^{\circ}+\mathrm{OH}^{-} \text {(Haber-Weiss reaction) }
\end{array}
$$

Fig. 2. Fenton and Haber-Weiss reactions are a source of oxidative stress. The generation of oxygen free radicals occurs first with the reduction of ferric to ferrous iron and then by the Fenton reaction with ferrous iron catalyzing the breakdown of hydrogen peroxide to hydroxyl radical and hydroxyl. The net reaction is termed the Haber-Weiss reaction.

orchestrate a careful balance of iron acquisition and binding to avoid injury while still effectively delivering iron to tissues as a critical nutrient.

Iron is acquired from the gut and absorbed through enterocytes. Through the portal system, iron is transported to the liver and then enters the circulation. TF is the plasma protein responsible for transporting iron in the plasma and then into the tissues. In addition to iron transport, TF has high affinity for free iron and functions as the body's natural iron chelator, effectively binding iron and preventing the formation of CFI. Iron is received by the tissues via the TF receptor. The largest consumer of iron in the body is the bone marrow where TF delivers iron via TF receptor for the production of erythrocytes. TF also delivers iron to tissues for the production of myoglobin, cytochromes, and other iron-containing enzymes.

In acute illness, iron homeostasis is often significantly disrupted such that CFI is produced and begins to contribute to tissue injury and pathologic effects. ${ }^{25}$ Many mechanisms contribute to an increase in CFI during acute illness, such as catecholamine surges, metabolic acidosis, tissue injury, hemolysis, and bleeding. ${ }^{26-30}$ Because CFI rapidly induces an increase in ROS, which causes more tissue injury leading to the release of cell-free heme and other iron-containing products, the liberation of $\mathrm{CFI}$ is self-sustaining and further exacerbates acute injury. ${ }^{25,31}$ Thus, CFI has been implicated as a critical injury pathway in many acute illnesses, such as myocardial infarction, sepsis, stroke, acute kidney injury (AKI), traumatic injuries, lung injury, stem cell

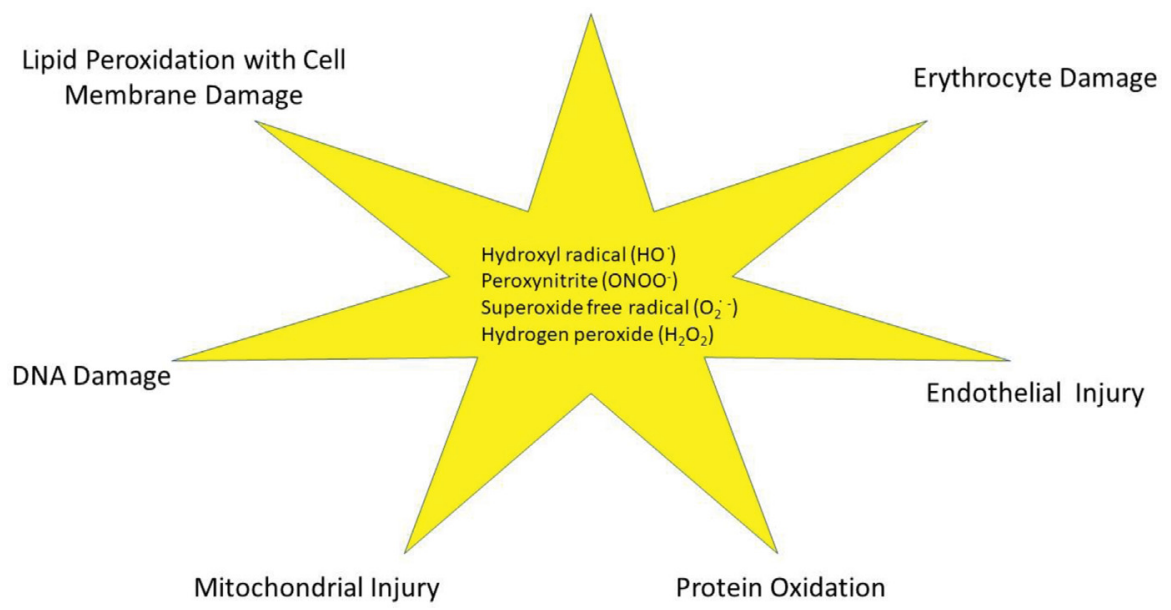

Fig. 3. Effects of reactive oxygen species. An excess of reactive oxygen species causes tissuespecific oxidative damage. Reactive oxygen species can damage DNA, proteins, and lipids, altering their structure and affecting function, ultimately contributing to various pathologies. 
transplantation, and reperfusion injury. ${ }^{27,30,32-38}$ The increase in CFI in acute illness is further exacerbated by the fact that iron is also a key nutrient for microorganisms that proliferate and induce virulence factors in the presence of iron. ${ }^{39,40}$ Thus, noninfectious acute injuries increase the risk of infection because of increased CFI, and infection-mediated injuries (ie, sepsis) are worsened by the increase in CFI.

The typical sequence of events is as follows. First, an injurious event causes a stress response. The classic fight or flight response is induced by increased sympathetic tone and a catecholamine surge. Catecholamines effectively cause the release of free-iron from hepatocytes and myocytes such that serum iron levels are increased rapidly. ${ }^{41}$ Although this response is likely adaptive (ie, increasing nutrient availability during stress), the increase in background iron is then exacerbated by the iron release that is caused by tissue injury from noninfectious or infectious insult. This rapid double-hit creates iron levels that exceed the binding capacity of TF leading to increased CFI levels thereby increasing ROS. During significant levels of injury, the induction of ROS can initiate a vicious cycle (see Fig. 3; Fig. 4).

The inflammatory response leads to hepcidin production and other acute-phase reactants to be produced that help ameliorate the iron-associated injury. Because hepcidin is induced by IL-6, many studies show that hepcidin is associated with worse outcomes and some have suggested that hepcidin has negative impacts. ${ }^{42,43}$ In the view of the authors, that is akin to judging firemen to be negative actors because more firemen are seen at larger fires. In the estimation of the authors, it is noteworthy that many of the acute-phase reactants are proteins that protect against ironassociated injury. For instance, the acute-phase proteins include hemopexin, haptoglobin, ferritin, lactoferrin, ceruloplasmin, NGAL, and hepcidin, all of which are involved in iron or iron-complexed macromolecule protective functions. ${ }^{22,44-50}$

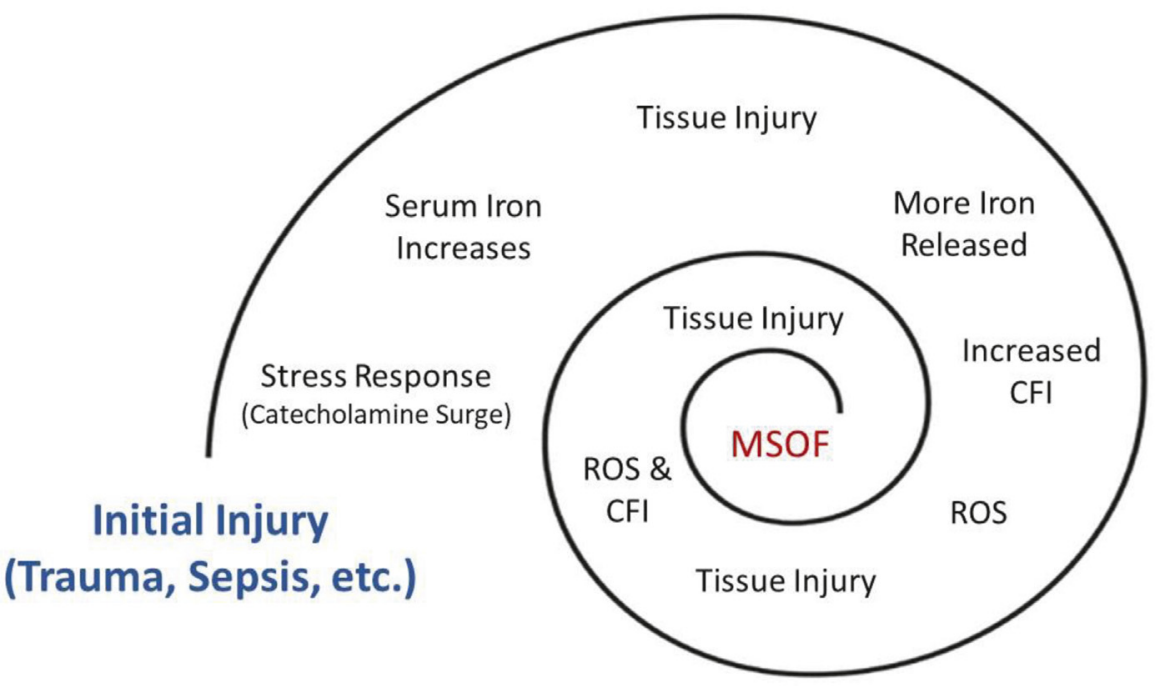

Fig. 4. Cycle of oxidative damage. An initial insult causes a stress response, which includes a surge of catecholamines that facilitates the release of iron from storage organs and increases serum iron. The dramatic increase of catalytic free iron participates in Fenton chemistry, generates reactive oxygen species, and causes tissue-specific oxidative damage. The tissue injury causes more iron to be released and the vicious cycle continues with the potential to result in multisystem organ failure. MSOF, multisystem organ failure. 
Initial placebo-controlled studies in healthy volunteers demonstrate that exogenous hepcidin induces a rapid increase in the acute-phase protein serum ferritin in addition to hypoferremia. ${ }^{51}$ The authors hypothesize that other iron-related acute-phase reactants are also increased by the administration of exogenous hepcidin. If this hypothesis is verified, exogenous hepcidin might be used to augment the natural iron injury defense system of the host to more rapidly and forcefully treat iron and cellfree hemoglobin-induced injury during acute illness. In essence, exogenous hepcidin may be a method to initiate a broad iron protective response that we denote as "siderotherapy."

\section{HEPCIDIN AS AN AGENT FOR SIDEROTHERAPY}

Hepcidin is the body's master regulator of iron and when induced, rapidly and effectively reduces plasma iron and initiates intracellular signaling for intracellular iron protective processes. Hepcidin induces hypoferremia by decreasing the TF saturation thereby activating the body's natural chelator. Hepcidin in the plasma causes the rapid degradation of ferroportin in macrophages in the liver, spleen, and plasma space. Under normal conditions, more than $90 \%$ of the daily iron supply is derived from macrophages. ${ }^{15,52}$ In addition, the degradation of ferroportin in enterocytes prevents further iron absorption in the gut. Thus, the supply of iron into the plasma is shut off. Because the bone marrow is in a constant state of iron uptake to generate erythrocytes to replace the senescent erythrocytes, the combination of bone marrow demand and reduced supply from hepcidin degradation of ferroportin results in a rapid drop in the serum iron and the TF saturation (see Fig. 1).

The pharmacodynamics of hepcidin show that parenteral hepcidin results in a rapid decrease in serum iron and TF saturation with a nadir at 8 to 12 hours. ${ }^{53}$ As such, hepcidin could have a role as a preventive agent and as a therapeutic agent after the acute injury has occurred.

In addition to inducing plasma hypoferremia and hyperferritinemia, hepcidin has been demonstrated in preclinical studies to induce intracellular ferritin, often referred to as $\mathrm{H}$-ferritin. ${ }^{38}$ Ferritin is a highly conserved protein across mammalian species and has two key functions. First, ferritin is effective at sequestering and storing iron in its nonreactive form. Second, ferritin is a ferroxidase and rapidly converts ferrous ( $\mathrm{Fe}^{2+}$, very toxic) to ferric ( $\mathrm{Fe}^{3+}$, somewhat less toxic) iron. ${ }^{45,46}$ Ferric iron is rapidly loaded onto TF in the plasma and ferric iron is also sequestered into ferritin. ${ }^{46}$ In general, ferritin is made up of light $(L)$ and heavy $(H)$ subunits. In the plasma, the ferritin has a higher proportion of L-subunits, whereas intracellular ferritin is largely made of $\mathrm{H}$ subunits. Both $\mathrm{L}$-rich and $\mathrm{H}$-rich ferritins have ferroxidase activity. L-rich ferritins are slower to incorporate iron but can hold more, whereas $\mathrm{H}$-rich ferritins can rapidly incorporate iron but can store less. ${ }^{45,46}$

The degradation of ferroportin by hepcidin prevents iron egress from the cell, thus increasing the intracellular iron concentration. Increased intracellular iron levels sets off a series of events that signals the cell to detoxify and rapidly store iron. The presence of $\mathrm{H}$-rich ferritin is cytoprotective and allows cells to withstand injury, making them more resistant to apoptosis and necroptosis. ${ }^{38,54}$ Preclinical studies demonstrate that induction or administration of hepcidin improves survival and decreases injury scores in preclinical models of AKI. ${ }^{38,55}$

An easy way to understand the effects of hepcidin is seen in the syndrome known as anemia of chronic disease, which is caused by high serum levels of hepcidin. ${ }^{56}$ This syndrome is most commonly encountered in patients with chronic inflammation (ie, uremia, rheumatoid arthritis, osteomyelitis). Although classically seen in inflammation, 
this syndrome almost certainly evolved as an adaptive response to chronic infection. The host's response to a chronic infection is to inflame and to make iron as scarce as possible because microbes require iron to survive. The syndrome is characterized by hypoferremia, low TF saturation, high ferritin, decreased iron absorption, and high levels of macrophage iron. ${ }^{56}$ This syndrome manifests as a microcytic anemia but is more accurately described as an iron scarcity syndrome. Thus, increasing levels of hepcidin may accelerate the body's conversion to an iron-scarce environment during acute injury to protect the host from CFI-induced damage with various acute diseases. If the level of injury during the acute insult is limited, the consequent inflammation may be reduced, and outcomes improved (see Fig. 4).

\title{
USE OF HEPCIDIN IN ACUTE ILLNESS
} Infectious Diseases

All eukaryotic and prokaryotic cells require iron to live, reproduce, and flourish. Bacteria, fungi, and parasites have all evolved strategies to acquire iron from the environment and/or the host to thrive. Certain infectious microbes are more vulnerable to the iron scarcity effects of hepcidin. The notion that hypoferremic interventions can improve outcomes in infected patients is not new. ${ }^{39}$ However, the treatments currently available to lower iron are all iron chelators. The main limitation of all the available ironchelators is that the chelator-iron complex may deliver iron to microbes and exacerbate the infection. ${ }^{39,57}$ In addition, available iron chelators have significant toxicity, which includes but is not limited to neutropenia, kidney injury, and liver injury. Because hepcidin uses the body's natural hypoferremic pathways, we postulate that this approach would be safer and more effective than iron chelation therapy. This section reviews the various potential opportunities to use hepcidin as an adjuvant agent in the treatment of acute infections. Hepcidin itself has antimicrobial properties, but the most logical way to use hepcidin may be as adjuvant treatment alongside standard antimicrobials for the treatment of infectious disease.

\section{Enteric gram-negative bacilli}

Patients with iron overload, such as hemochromatosis, transfusional iron overload, and cirrhosis, are more susceptible to certain gram-negative bacilli, particularly siderophilic bacilli (Box 2). ${ }^{58,59}$ Deadly infections with siderophilic gram-negative bacilli, such as Yersinia enterocolitica, Vibrio vulnificus, and Klebsiella pneumoniae, have been reported in patients with iron overload. ${ }^{60,61}$ Nonclinical models of these infectious agents demonstrate that increased CFI increases the lethality of these

\author{
Box 2 \\ Siderophilic pathogens \\ Vibrio vulnificus \\ Listeria monocytogenes \\ Yersenia enterocolitica \\ Salmonella enterica \\ Klebsiella pneumoniae \\ Escherichia coli \\ Rhizopus arrhizus \\ Mucor
}


infections. ${ }^{62-64}$ In these models, hepcidin agonists rapidly decreased iron levels and improved survival. ${ }^{62-64}$ Hepcidin added to standard therapy has the potential to improve outcomes in these infections particularly among those patients with iron overload.

It should be noted that hepcidin therapy added to standard antimicrobial therapy for bacterial infections may be useful. The early phase of injury and stress causes increased levels of endogenous catecholamines, which facilitates the release of iron. Because exogenous catecholamines (ie, norepinephrine, dopamine) are part of the standard care of patients with septic shock it is likely that clinicians may unwittingly worsen the infection by providing more iron via catecholamine therapy. ${ }^{65}$ Thus, adding hepcidin therapy when patients first present to medical care, before or soon after the initiation of antimicrobial and/or catecholamine therapy, may prove beneficial.

Another potential use for hepcidin therapy is in patients who are infected with highly resistant gram-negative bacilli, such as Acinetobacter baumannii, K. pneumoniae, and other similar siderophilic bacilli. There are two key concepts that inform this hypothesis. First, the acquisition of resistance for a micro-organism is not free. Resistance involves altering the normal intracellular machinery of a bacterium and hence the bacterium must pay a fitness cost for this resistance attribute. ${ }^{66}$ This concept of fitness was discovered because of the observation that when resistant microorganisms (bacteria, fungi, or parasites) are moved into an environment without antibiotic pressure, they are outcompeted by nonresistant microbes that can acquire nutrients and divide faster unencumbered by resistance machinery. ${ }^{66}$ Second, in animal models of resistant bacteria, the addition of antimicrobial agents improves survival even if the microbe is resistant to that agent. ${ }^{67,68}$ Some investigators have postulated that even if the microbe is resistant, the presence of the antimicrobial in the environment forces the pathogen to behave in a way that either lessens virulence or allows for a more efficient immune response. ${ }^{39}$ We postulate that because resistant organisms are less fit, they may be more susceptible to an approach that rapidly removes a critical nutrient from their environment. Moreover, because none of the available antibiotics or other antimicrobial therapeutics target iron acquisition, induction of hypoferremia may be a thoughtful vulnerability to exploit. Because hepcidin induces extracellular and intracellular iron scarcity, the addition of robust hypoferremia may be particularly effective for patients infected with resistant organisms who have few alternative therapies available.

\section{Fungal infections}

Although all fungi require iron to thrive, certain pathogenic fungi are more siderophilic and thus may be more sensitive to hypoferremic interventions. Preclinical and clinical studies demonstrate that iron overload favors the development of fungal infections and may increase pathogen virulence. ${ }^{69-71}$

Patients who undergo myeloablative or bone marrow conditioning therapies are at risk for iron overload because these therapies decrease the demand from the bone marrow. As such, the continued egress of iron from macrophages and splenocytes from erythrophagocytosis exceeds that bone marrow uptake, which results in high TF saturation and increased CFI (see Fig. 1). ${ }^{37,72}$ These patients are susceptible to infection in part because of increased iron availability and immune dysfunction, notably the presence of neutropenia. Consequently, these patients tend to become infected with siderophilic bacteria and fungi. The fungi that are most common and pathogenic are Candida spp, Aspergillus, and Mucor. Although iron-chelation therapy has not shown any benefit in clinical studies of fungal infection, this type of hypoferremic therapy is confounded by the potential enhanced delivery of iron to the 
microbes. ${ }^{73}$ For patients with iron overload manifested by an increased TF saturation, hepcidin-induced hypoferremia may have an important role as a prophylactic agent and may also have a role as a therapeutic in patients who are infected.

\section{Malaria}

A complete discussion about hepcidin and malaria is beyond the scope of this article. In brief, hepcidin has potentially three key roles in the treatment of malaria. First is as a prophylaxis of liver stage malaria. Plasmodium species have a complex life cycle and after being bitten by a mosquito, plasmodium schizonts must first invade the liver and go through a liver stage before being able to infect the blood and continue the infection. ${ }^{74}$ A nonclinical study suggests that prior ongoing malaria infection may cause enough hepcidin production to prevent coinfections with other strains of malaria. ${ }^{75}$ This is likely because hepcidin generates a brisk hypoferremic effect within hepatocytes thereby making them less conducive to infection and propagation. We postulate that because of this effect, exogenous hepcidin may have a role as a prophylactic medication for infections with plasmodium species especially for people traveling to areas with endemic malaria. Hepcidin could also be used to facilitate live-schizont vaccination regimens. ${ }^{76}$ Specifically, live-schizont vaccine therapies are predicated on creating a moat around the liver preventing systemic infection and allowing the immune system to become activated and develop immunity. However, this approach would not work for forms of malaria that develop chronic liver stage infection. Exogenous hepcidin given before Plasmodium vivax or Plasmodium falciparum schizont inoculation may prevent liver stage infection and allow the immune system to develop durable immunity.

A second opportunity for hepcidin involves the treatment of the liver stage of malaria. Certain plasmodium species have hypnozoites, which are forms of malaria that can lay dormant in the liver for years and then reactivate. The authors propose that hepcidin may have capacity as a drug to help eradicate liver stage plasmodium species similar to the use of the drug tafenoquine. ${ }^{77}$ Tafenoquine has been shown to be effective in radical cure of $P$ vivax, but tafenoquine is associated with toxicity. We suggest that hepcidin may be used in conjunction with tafenoquine allowing for a decreased dose that may improve synergy and decrease toxicity.

The third potential role for hepcidin in malaria is related to resistance. Plasmodia species that infect humans are efficient at developing resistance. New strains of plasmodia have emerged that are resistant to artemisinin and quinine antimicrobials. Because plasmodia species also pay a fitness cost for the acquisition of resistance, we believe that the addition of hepcidin to current antimalarial regimens may restore artemisinin and/or quinine sensitivity. ${ }^{78,79}$

\section{Sepsis}

Preclinical studies assessing a multitude of sepsis models indicate that hepcidin is protective. ${ }^{36,80,81}$ Although most studies identify the reduction of CFI as the key mechanism for improving outcomes, particularly in models that assess siderophilic or enteric organisms, it seems that the protective mechanism is not exclusively related to iron. Other nonclinical studies show that hepcidin also has effects on immune cells. ${ }^{82,83}$ One example is that hepcidin favors the M2 over M1 polarization of macrophages, which may be a more optimal distribution of macrophage phenotype in endorgan outcomes. ${ }^{84,85}$ The non-iron immune cell effects of hepcidin are reviewed extensively elsewhere and are beyond the scope of this review. ${ }^{82,83}$ Nonetheless, the key message is that hepcidin evolved as the key regulator of iron to help protect against infection. Therefore, it is logical that hepcidin would have other pleiotropic effects that augment the host's ability to respond to infection. ${ }^{86}$ 


\section{Noninfectious Diseases}

\section{Acute kidney injury}

Iron-mediated oxidative stress is implicated in the pathogenesis of renal ischemia reperfusion injury ([IRI] a major cause of $\mathrm{AKI})$, and multiple animal models have demonstrated that catalytic iron is a key mediator of AKI. ${ }^{33,38,87}$ In disease states, such as acute kidney diseases, after IRI, sepsis, and myocardial infarction, CFI is increased. ${ }^{35}$ This is caused by a decrease in $\mathrm{pH}$ or free radical-induced reduction of iron that causes dissociation of bound intracellular iron, yielding free iron that can serve as a catalyst for vulnerable situations of oxidative stress. ${ }^{88}$ During operations that require cardiopulmonary bypass (CPB; which is inherently a high CFI environment caused by surgical stress) extracorporeally circulated blood exposed to nonphysiologic surfaces and shear forces may injure red blood cells, releasing free hemoglobin and free iron; association with adverse postoperative outcomes is observed with $\mathrm{CFI} .{ }^{33}$ Moreover, in patients who are undergoing surgery requiring CPB, TF saturation routinely exceeds $50 \%$ during surgery and consequently plasma CFI increases, which is strongly linked to $\mathrm{AKI}^{29,33,38,87,89}$ In addition, significant elevations in CFI, but not other iron parameters, have been observed in patients after cardiac surgery and were directly associated with CPB time and number of packed red blood cell transfusions. ${ }^{33}$

Interventional studies to reduce CFI levels are hypothesized to prevent $\mathrm{AKI}^{3}{ }^{33,38}$ Endogenous hepcidin regulates iron absorption and recycling, provides protection from reactive iron species, and is a biomarker for iron-related pathophysiology.

It has been observed in murine models that hepcidin is freely filtered by the glomerulus and reabsorbed in the proximal tubule cells by megalin-dependent endocytosis. ${ }^{55,90}$ In animal models of $\mathrm{AKI}, \mathrm{CFI}$ has been implicated in nephrotoxicity resulting from a wide range of insults including ischemia/reperfusion, aminoglycosides, rhabdomyolysis, and hemoglobinuria. Iron chelators, such as deferiprone and deferoxamine, have been demonstrated to protect against experimental cardiac IRI in diverse animal models, establishing a cause and effect relationship. ${ }^{35}$ Animal models have also shown that hepcidin-deficient mice demonstrated increased susceptibility to IRI compared with wild-type mice and that reconstitution with exogenous hepcidin induced hepatic iron sequestration; attenuated reduction of renal tissue ferritin; and reduced oxidative stress, apoptosis, inflammation, and tubular injury. Similar models have shown that hepcidin treatment restores iron homeostasis and reduces inflammation to mediate protection in renal IRI. ${ }^{38}$

Overall, the authors suggest that pretreatment with hepcidin before surgeries requiring $\mathrm{CPB}$ may have five key effects ${ }^{38}$ :

1. Prevents initial surge of iron from the spleen and liver via degradation of ferroportin in macrophages, hepatocytes, and splenocytes.

2. Reduction of TF saturation in preparation for CPB, thereby forming an iron buffer (TF is the body's natural chelator) that can accommodate the free iron liberated by CPB and transfusion.

3. Induction of intracellular $\mathrm{H}$-ferritin in the proximal convoluted tubule cells from filtered hepcidin uptake before cardiopulmonary surgeries requiring CPB, thereby inducing a cytoprotective response in the kidney.

4. Increase in serum ferritin providing more rapid loading of free iron onto TF and providing a sink for free iron.

5. Induction of a more favorable immune response.

Taken together, the central biologic role of hepcidin in iron metabolism and the possible importance of iron in the pathogenesis of CPB-associated AKI identify 
hepcidin as a potential treatment in preventing $\mathrm{AKI}$ in patients undergoing surgery requiring $\mathrm{CPB}$.

\section{Trauma and transfusion}

Patients with severe trauma often require massive blood transfusion. ${ }^{91}$ Because banked blood becomes more fragile over time when stored, a significant proportion of the transfused blood is removed from the circulation rapidly and undergoes extravascular hemolysis. ${ }^{92}$ When this process occurs, the iron from the phagocytosed red cells is released into the bloodstream thereby increasing CFI. ${ }^{93}$ This release of free iron after transfusion is consistent and even occurs in healthy volunteers receiving autologous blood. ${ }^{94}$ The increased $\mathrm{CFI}$ environment seen in patients with trauma receiving massive blood transfusion is exacerbated because many trauma patients require catecholamine infusions (which increase serum iron) and suffer severe anemia from hemorrhage, which leads to the release of erythropoietin, which suppresses hepcidin generation thereby increasing CFI. ${ }^{13,26,41,95}$ As a consequence, severe trauma is often exacerbated by high levels of $\mathrm{CFI}$. Thus, patients with trauma requiring massive blood transfusion leading to high-levels of CFI may benefit from hepcidin-induced hypoferremia. Ideally, hepcidin therapy would be initiated immediately on presentation to hospital to maximize the potential benefits of counteracting the increased CFI seen in trauma patients requiring massive blood transfusion.

\section{Cardioprotection}

Iron plays a key role in the injury associated with reperfusion. ${ }^{96}$ Thus, there have been multiple investigations assessing hypoferremic modalities in myocardial infarction and cardioprotection. Preclinical studies indicate that myocardial reperfusion injury is ameliorated by hypoferremia induced by iron chelation therapy. ${ }^{97-99}$ Clinical trials in humans after bypass surgery supported these findings by demonstrating that treatment with the iron chelator deferoxamine ameliorated lipid peroxidation and protected the myocardium from reperfusion injury. ${ }^{100,101}$ The addition of deferoxamine to vasoplegia solution has been shown to offer better cardioprotection in large mammal studies of CPB. ${ }^{102}$ In a large cohort trial, higher levels of CFI were associated with increased risk of death in patients with acute coronary syndrome. ${ }^{27}$

In aggregate, these data suggest that the safe and rapid induction of hypoferremia by hepcidin may protect against reperfusion injury and offer myocardial protection in acute coronary syndrome and CPB.

\section{Liver disease}

The source of plasma hepcidin is largely liver-derived and patients with advanced liver disease lose synthetic function of liver proteins. ${ }^{103,104}$ The loss of synthetic function results in low plasma levels of TF and low levels of hepcidin, which leads to increased CFI. ${ }^{103,104}$ Iron overloaded states in nonalcoholic fatty liver disease, indicated by elevated serum ferritin, are associated with increased hepatic iron deposition, advancement of hepatic fibrosis, and a diagnosis of nonalcoholic steatohepatitis. ${ }^{105}$ As such, patients with severe liver disease often suffer from complications that are found in patients with chronic iron overload. As an example, patients with cirrhosis are susceptible to siderophilic gram-negative bacilli, such as $V$ vulnificus and enteric bacilli. ${ }^{106,107}$ As evidence of excess iron and risk, cohort studies have shown that dysregulated iron homeostasis is associated with multiorgan failure and early mortality in acute-on-chronic liver failure. ${ }^{104,108}$ The main risk factors identified were low serum TF levels, elevated TF saturation, and decreased hepcidin levels. An environment of increased CFI makes the host more vulnerable to infection and makes the risk of severe ROS associated with infection more likely. 
Exogenous hepcidin hypoferremic effects could be used for prevention in patients with advanced liver disease and might also have an adjunctive role in treating infections in patients with cirrhosis, particularly those caused by siderophilic bacilli.

\section{SUMMARY}

Acute diseases tend to worsen when CFI is increased. Iron chelators can induce rapid hypoferremia but have significant toxicity. Hepcidin, the body's master regulator of iron, has the capacity to induce hypoferremia, initiate cytoprotective pathways, and favorably affect the immune response. Thus, exogenous hepcidin may have a role in the treatment of acute diseases associated with elevated CFI.

\section{REFERENCES}

1. Kell DB. Iron behaving badly: inappropriate iron chelation as a major contributor to the aetiology of vascular and other progressive inflammatory and degenerative diseases. BMC Med Genomics 2009;2:2.

2. Pigeon C, Ilyin G, Courselaud B, et al. A new mouse liver-specific gene, encoding a protein homologous to human antimicrobial peptide hepcidin, is overexpressed during iron overload. J Biol Chem 2001;276(11):7811-9.

3. Nicolas G, Bennoun M, Devaux I, et al. Lack of hepcidin gene expression and severe tissue iron overload in upstream stimulatory factor 2 (USF2) knockout mice. PNAS 2001;98(15):6.

4. Nemeth E, Tuttle MS, Powelson J, et al. Hepcidin regulates cellular iron efflux by binding to ferroportin and inducing its internalization. Science 2004;306:4.

5. Krause A, Neitz S, Magert H, et al. LEAP-1, a novel highly disulfide-bonded human peptide, exhibits antimicrobial activity. FEBS Lett 2000;480:4.

6. Park $\mathrm{CH}$, Valore EV, Waring AJ, et al. Hepcidin, a urinary antimicrobial peptide synthesized in the liver. J Biol Chem 2001;276(11):7806-10.

7. Roetto A, Papanikolaou G, Politou M, et al. Mutant antimicrobial peptide hepcidin is associated with severe juvenile hemochromatosis. Nat Genet 2003;33(1):21-2.

8. Ganz T. Hepcidin and iron regulation, 10 years later. Blood 2011;117(17): 4425-33.

9. Ganz T, Nemeth E. Iron metabolism: interactions with normal and disordered erythropoiesis. Cold Spring Harb Perspect Med 2012;2(5):a011668.

10. Nemeth E, Ganz T. Regulation of iron metabolism by hepcidin. Annu Rev Nutr 2006;26:323-42.

11. Nicolas G, Bennoun M, Porteu A, et al. Severe iron deficiency anemia in transgenic mice expressing liver hepcidin. Proc Natl Acad Sci U S A 2002;99(7): 4596-601.

12. Weinstein DA, Roy CN, Fleming MD, et al. Inappropriate expression of hepcidin is associated with iron refractory anemia: implications for the anemia of chronic disease. Blood 2002;100(10):3776-81.

13. Nicolas G, Chauvet C, Viatte L, et al. The gene encoding the iron regulatory peptide hepcidin is regulated by anemia, hypoxia, and inflammation. J Clin Invest 2002;110(7):1037-44.

14. D'Alessio F, Hentze MW, Muckenthaler MU. The hemochromatosis proteins HFE, TfR2, and HJV form a membrane-associated protein complex for hepcidin regulation. J Hepatol 2012;57(5):1052-60.

15. Hentze MW, Muckenthaler MU, Galy B, et al. Two to tango: regulation of mammalian iron metabolism. Cell 2010;142(1):24-38. 
16. Andriopoulos B Jr, Corradini E, Xia Y, et al. BMP6 is a key endogenous regulator of hepcidin expression and iron metabolism. Nat Genet 2009;41(4):482-7.

17. Babitt JL, Huang FW, Wrighting DM, et al. Bone morphogenetic protein signaling by hemojuvelin regulates hepcidin expression. Nat Genet 2006; 38(5):531-9.

18. Casanovas G, Mleczko-Sanecka K, Altamura S, et al. Bone morphogenetic protein (BMP)-responsive elements located in the proximal and distal hepcidin promoter are critical for its response to HJV/BMP/SMAD. J Mol Med (Berl) 2009; 87(5):471-80.

19. Du X, She E, Gelbart T, et al. The serine protease TMPRSS6 is required to sense iron deficiency. Science 2008;320(5879):5.

20. Silvestri L, Pagani A, Nai A, et al. The serine protease matriptase-2 (TMPRSS6) inhibits hepcidin activation by cleaving membrane hemojuvelin. Cell Metab 2008;8(6):502-11.

21. Wrighting DM, Andrews NC. Interleukin-6 induces hepcidin expression through STAT3. Blood 2006;108(9):3204-9.

22. Nemeth E, Rivera S, Gabayan V, et al. IL-6 mediates hypoferremia of inflammation by inducing the synthesis of the iron regulatory hormone hepcidin. J Clin Invest 2004;113(9):6.

23. Kehrer JP. The Haber-Weiss reaction and mechanisms of toxicity. Toxicology 2000;149(1):43-50.

24. Wardman P, Candeias LP. Fenton chemistry: an introduction. Radiat Res 1996; 145(5):523-31.

25. Halliwell B, Gutteridge JM. Role of free radicals and catalytic metal ions in human disease: an overview. Methods Enzymol 1990;186:1-85.

26. Sandrini SM, Shergill R, Woodward J, et al. Elucidation of the mechanism by which catecholamine stress hormones liberate iron from the innate immune defense proteins transferrin and lactoferrin. J Bacteriol 2010;192(2):587-94.

27. Lele SS, Mukhopadhyay BN, Mardikar MM, et al. Impact of catalytic iron on mortality in patients with acute coronary syndrome exposed to iodinated radiocontrast: the Iscom Study. Am Heart J 2013;165(5):744-51.

28. Baliga R, Zhang Z, Baliga M, et al. Evidence for cytochrome P-450 as a source of catalytic iron in myoglobinuric acute renal failure. Kidney Int 1996;49(2): 362-9.

29. Pepper JR, Mumby S, Gutteridge JM. Sequential oxidative damage, and changes in iron-binding and iron-oxidising plasma antioxidants during cardiopulmonary bypass surgery. Free Radic Res 1994;21(6):377-85.

30. Fuernau G, Traeder F, Lele SS, et al. Catalytic iron in acute myocardial infarction complicated by cardiogenic shock: a biomarker substudy of the IABP-SHOCK II-trial. Int J Cardiol 2017;227:83-8.

31. Balla G, Vercellotti GM, Muller-Eberhard U, et al. Exposure of endothelial cells to free heme potentiates damage mediated by granulocytes and toxic oxygen species. Lab Invest 1991;64(5):648-55.

32. Lagan AL, Melley DD, Evans TW, et al. Pathogenesis of the systemic inflammatory syndrome and acute lung injury: role of iron mobilization and decompartmentalization. Am J Physiol Lung Cell Mol Physiol 2008;294(2):L161-74.

33. Leaf DE, Rajapurkar M, Lele SS, et al. Increased plasma catalytic iron in patients may mediate acute kidney injury and death following cardiac surgery. Kidney Int 2015;87(5):1046-54. 
34. Haase M, Bellomo R, Haase-Fielitz A. Novel biomarkers, oxidative stress, and the role of labile iron toxicity in cardiopulmonary bypass-associated acute kidney injury. J Am Coll Cardiol 2010;55(19):2024-33.

35. Shah SV, Rajapurkar MM, Baliga R. The role of catalytic iron in acute kidney injury. Clin J Am Soc Nephrol 2011;6(10):2329-31.

36. Chen QX, Song SW, Chen QH, et al. Silencing airway epithelial cell-derived hepcidin exacerbates sepsis induced acute lung injury. Crit Care 2014;18(4):470.

37. Pullarkat V. Iron overload in patients undergoing hematopoietic stem cell transplantation. Adv Hematol 2010;2010 [pii:345756].

38. Scindia $Y$, Dey $P$, Thirunagari $A$, et al. Hepcidin mitigates renal ischemiareperfusion injury by modulating systemic iron homeostasis. J Am Soc Nephrol 2015;26(11):2800-14.

39. Cassat JE, Skaar EP. Iron in infection and immunity. Cell Host Microbe 2013; 13(5):509-19.

40. Hood MI, Skaar EP. Nutritional immunity: transition metals at the pathogen-host interface. Nat Rev Microbiol 2012;10(8):525-37.

41. Tapryal N, Vivek GV, Mukhopadhyay CK. Catecholamine stress hormones regulate cellular iron homeostasis by a posttranscriptional mechanism mediated by iron regulatory protein: implication in energy homeostasis. J Biol Chem 2015; 290(12):7634-46.

42. Xiong XY, Liu L, Wang FX, et al. Toll-like receptor 4/MyD88-mediated signaling of hepcidin expression causing brain iron accumulation, oxidative injury, and cognitive impairment after intracerebral hemorrhage. Circulation 2016;134(14): 1025-38.

43. Wagner M, Ashby DR, Kurtz C, et al. Hepcidin-25 in diabetic chronic kidney disease is predictive for mortality and progression to end stage renal disease. PLoS One 2015;10(4):e0123072.

44. Smith A, McCulloh RJ. Hemopexin and haptoglobin: allies against heme toxicity from hemoglobin not contenders. Front Physiol 2015;6:187.

45. Mehlenbacher M, Poli M, Arosio P, et al. Iron oxidation and core formation in recombinant heteropolymeric human ferritins. Biochemistry 2017;56(30):3900-12.

46. Harrison PM, Arosio P. The ferritins: molecular properties, iron storage function and cellular regulation. Biochim Biophys Acta 1996;1275(3):161-203.

47. Baker HM, Baker EN. A structural perspective on lactoferrin function. Biochem Cell Biol 2012;90(3):320-8.

48. Attieh ZK, Mukhopadhyay CK, Seshadri V, et al. Ceruloplasmin ferroxidase activity stimulates cellular iron uptake by a trivalent cation-specific transport mechanism. J Biol Chem 1999;274(2):1116-23.

49. Bao G, Clifton M, Hoette TM, et al. Iron traffics in circulation bound to a siderocalin (NGAL)-catechol complex. Nat Chem Biol 2010;6(8):602-9.

50. Goetz DH, Holmes MA, Borregaard N, et al. The neutrophil lipocalin NGAL is a bacteriostatic agent that interferes with siderophore-mediated iron acquisition. Mol Cell 2002;10(5):1033-43.

51. A phase 1, randomized, double-blind, two-arm, placebo-controlled, single and multiple dose escalation study to assess the safety and tolerability of LJPC-401 in healthy adults. 2018. LJ401-NHV02. Available at: https://learningcenter. ehaweb.org/eha/2018/stockholm/214475/vip.viprakasit.a. phase.1.open-label. study.to. determine.the. safety.tolerability.html?f=topic $=1574^{*}$ media $=3$.

52. Pantopoulos K, Porwal SK, Tartakoff A, et al. Mechanisms of mammalian iron homeostasis. Biochemistry 2012;51(29):5705-24. 
53. Yeager D, Piga A, Lai A, et al. A phase 1, placebo-controlled study to determine the safety, tolerability, and pharmacokinetics of escalating subcutaneous doses of LJPC-401 (synthetic human hepcidin) in healthy adults. 23rd European Hematology Association Congress. Stockholm, June 15, 2018.

54. Zarjou A, Bolisetty S, Joseph R, et al. Proximal tubule $\mathrm{H}$-ferritin mediates iron trafficking in acute kidney injury. J Clin Invest 2013;123(10):4423-34.

55. van Swelm RP, Wetzels JF, Verweij VG, et al. Renal handling of circulating and renal-synthesized hepcidin and its protective effects against hemoglobinmediated kidney injury. J Am Soc Nephrol 2016;27(9):2720-32.

56. Weiss G, Goodnough LT. Anemia of chronic disease. N Engl J Med 2005; 352(10): 1011-23.

57. Kim CM, Park YJ, Shin SH. A widespread deferoxamine-mediated iron-uptake system in Vibrio vulnificus. J Infect Dis 2007;196(10):1537-45.

58. Casu C, Nemeth E, Rivella S. Hepcidin agonists as therapeutic tools. Blood 2018;131(16):1790-4.

59. Khan FA, Fisher MA, Khakoo RA. Association of hemochromatosis with infectious diseases: expanding spectrum. Int J Infect Dis 2007;11(6):482-7.

60. Gerhard GS, Levin KA, Price Goldstein J, et al. Vibrio vulnificus septicemia in a patient with the hemochromatosis HFE C282Y mutation. Arch Pathol Lab Med 2001;125(8):1107-9.

61. Chung BH, Ha SY, Chan GC, et al. Klebsiella infection in patients with thalassemia. Clin Infect Dis 2003;36(5):575-9.

62. Stefanova D, Raychev A, Arezes J, et al. Endogenous hepcidin and its agonist mediate resistance to selected infections by clearing non-transferrin-bound iron. Blood 2017;130(3):245-57.

63. Arezes J, Jung G, Gabayan V, et al. Hepcidin-induced hypoferremia is a critical host defense mechanism against the siderophilic bacterium Vibrio vulnificus. Cell Host Microbe 2015;17(1):47-57.

64. Michels KR, Zhang Z, Bettina AM, et al. Hepcidin-mediated iron sequestration protects against bacterial dissemination during pneumonia. JCl Insight 2017; 2(6):e92002.

65. Lyte M, Freestone PP, Neal CP, et al. Stimulation of Staphylococcus epidermidis growth and biofilm formation by catecholamine inotropes. Lancet 2003; 361(9352): 130-5.

66. Andersson DI, Hughes D. Antibiotic resistance and its cost: is it possible to reverse resistance? Nat Rev Microbiol 2010;8(4):260-71.

67. McPherson CJ, Aschenbrenner LM, Lacey BM, et al. Clinically relevant gramnegative resistance mechanisms have no effect on the efficacy of $\mathrm{MC}-1$, a novel siderophore-conjugated monocarbam. Antimicrob Agents Chemother 2012; 56(12):6334-42.

68. Pramanik A, Stroeher UH, Krejci J, et al. Albomycin is an effective antibiotic, as exemplified with Yersinia enterocolitica and Streptococcus pneumoniae. Int J Med Microbiol 2007;297(6):459-69.

69. Saikia S, Oliveira D, Hu G, et al. Role of ferric reductases in iron acquisition and virulence in the fungal pathogen Cryptococcus neoformans. Infect Immun 2014; 82(2):839-50.

70. Iglesias-Osma C, Gonzalez-Villaron L, San Miguel J, et al. Iron metabolism and fungal infections in patients with haematological malignancies. J Clin Pathol 1995;48(3):3. 
71. Alexander J, Limaye AP, Ko CW, et al. Association of hepatic iron overload with invasive fungal infection in liver transplant recipients. Liver Transpl 2006;12(12): 1799-804.

72. Sahlstedt L, von Bonsdorff L, Ebeling F, et al. Non-transferrin-bound iron in haematological patients during chemotherapy and conditioning for autologous stem cell transplantation. Eur J Haematol 2009;83(5):455-9.

73. Spellberg B, Ibrahim AS, Chin-Hong PV, et al. The Deferasirox-AmBisome Therapy for Mucormycosis (DEFEAT Mucor) study: a randomized, double-blinded, placebo-controlled trial. J Antimicrob Chemother 2012;67(3):715-22.

74. Prudencio M, Rodriguez A, Mota MM. The silent path to thousands of merozoites: the Plasmodium liver stage. Nat Rev Microbiol 2006;4(11):849-56.

75. Portugal S, Carret C, Recker M, et al. Host-mediated regulation of superinfection in malaria. Nat Med 2011;17(6):732-7.

76. Mordmuller B, Surat G, Lagler H, et al. Sterile protection against human malaria by chemoattenuated PfSPZ vaccine. Nature 2017;542(7642):445-9.

77. Rajapakse S, Rodrigo C, Fernando SD. Tafenoquine for preventing relapse in people with Plasmodium vivax malaria. Cochrane Database Syst Rev 2015;(4):CD010458.

78. Rosenthal PJ. The interplay between drug resistance and fitness in malaria parasites. Mol Microbiol 2013;89(6):1025-38.

79. Spottiswoode N, Duffy PE, Drakesmith H. Iron, anemia and hepcidin in malaria. Front Pharmacol 2014;5:125.

80. Stefanova D, Raychev A, Deville J, et al. Hepcidin protects against lethal Escherichia coli sepsis in mice inoculated with isolates from septic patients. Infect Immun 2018;86(7):12.

81. Zeng $C$, Chen Q, Zhang K, et al. Hepatic hepcidin protects against polymicrobial sepsis in mice by regulating host iron status. Anesthesiology 2015;122(2): 374-86.

82. Pagani A, Nai A, Corna G, et al. Low hepcidin accounts for the proinflammatory status associated with iron deficiency. Blood 2011;118(3):736-46.

83. Riba M, Rausa M, Sorosina M, et al. A strong anti-inflammatory signature revealed by liver transcription profiling of Tmprss6-/- mice. PLoS One 2013;8(7): e69694.

84. Agoro R, Taleb M, Quesniaux VFJ, et al. Cell iron status influences macrophage polarization. PLoS One 2018;13(5):e0196921.

85. Martinez FO, Gordon S. The M1 and M2 paradigm of macrophage activation: time for reassessment. F1000prime Rep 2014;6:13.

86. Michels K, Nemeth E, Ganz T, et al. Hepcidin and host defense against infectious diseases. PLoS Pathog 2015;11(8):e1004998.

87. Walker VJ, Agarwal A. Targeting iron homeostasis in acute kidney injury. Semin Nephrol 2016;36(1):62-70.

88. Martines AM, Masereeuw R, Tjalsma H, et al. Iron metabolism in the pathogenesis of iron-induced kidney injury. Nat Rev Nephrol 2013;9(7):385-98.

89. Pepper JR, Mumby S, Gutteridge JM. Blood cardioplegia increases plasma iron overload and thiol levels during cardiopulmonary bypass. Ann Thorac Surg 1995;60(6):1735-40.

90. Peters HP, Laarakkers CM, Pickkers P, et al. Tubular reabsorption and local production of urine hepcidin-25. BMC Nephrol 2013;14:70.

91. Como JJ, Dutton RP, Scalea TM, et al. Blood transfusion rates in the care of acute trauma. Transfusion 2004;44(6):809-13. 
92. Rapido F, Brittenham GM, Bandyopadhyay S, et al. Prolonged red cell storage before transfusion increases extravascular hemolysis. J Clin Invest 2017;127(1): 375-82.

93. Brittenham GM. Iron-chelating therapy for transfusional iron overload. N Engl J Med 2011;364(2):146-56.

94. Hod EA, Brittenham GM, Billote GB, et al. Transfusion of human volunteers with older, stored red blood cells produces extravascular hemolysis and circulating non-transferrin-bound iron. Blood 2011;118(25):6675-82.

95. Pak M, Lopez MA, Gabayan V, et al. Suppression of hepcidin during anemia requires erythropoietic activity. Blood 2006;108(12):3730-5.

96. Halliwell B. Superoxide, iron, vascular endothelium and reperfusion injury. Free Radic Res Commun 1989;5(6):315-8.

97. Ambrosio G, Zweier JL, Jacobus WE, et al. Improvement of postischemic myocardial function and metabolism induced by administration of deferoxamine at the time of reflow: the role of iron in the pathogenesis of reperfusion injury. Circulation 1987;76(4):906-15.

98. van der Kraaij AM, Mostert LJ, van Eijk HG, et al. Iron-load increases the susceptibility of rat hearts to oxygen reperfusion damage. Protection by the antioxidant (+)-cyanidanol-3 and deferoxamine. Circulation 1988;78(2):442-9.

99. Williams RE, Zweier JL, Flaherty JT. Treatment with deferoxamine during ischemia improves functional and metabolic recovery and reduces reperfusion-induced oxygen radical generation in rabbit hearts. Circulation 1991;83(3):1006-14.

100. Paraskevaidis IA, Iliodromitis EK, Vlahakos D, et al. Deferoxamine infusion during coronary artery bypass grafting ameliorates lipid peroxidation and protects the myocardium against reperfusion injury: immediate and long-term significance. Eur Heart J 2005;26(3):263-70.

101. Ferreira R, Burgos M, Milei J, et al. Effect of supplementing cardioplegic solution with deferoxamine on reperfused human myocardium. J Thorac Cardiovasc Surg 1990;100(5):708-14.

102. Veres G, Radovits T, Merkely B, et al. Custodiol-N, the novel cardioplegic solution reduces ischemia/reperfusion injury after cardiopulmonary bypass. J Cardiothorac Surg 2015;10:27.

103. Tan TC, Crawford DH, Franklin ME, et al. The serum hepcidin:ferritin ratio is a potential biomarker for cirrhosis. Liver Int 2012;32(9):1391-9.

104. Bruns T, Nuraldeen R, Mai M, et al. Low serum transferrin correlates with acuteon-chronic organ failure and indicates short-term mortality in decompensated cirrhosis. Liver Int 2017;37(2):232-41.

105. Kowdley KV, Belt P, Wilson LA, et al. Serum ferritin is an independent predictor of histologic severity and advanced fibrosis in patients with nonalcoholic fatty liver disease. Hepatology 2012;55(1):77-85.

106. Ascione T, Di Flumeri $G$, Boccia $G$, et al. Infections in patients affected by liver cirrhosis: an update. Infez Med 2017;25(2):91-7.

107. Haq SM, Dayal HH. Chronic liver disease and consumption of raw oysters: a potentially lethal combination. A review of Vibrio vulnificus septicemia. Am J Gastroenterol 2005;100(5):1195-9.

108. Maras JS, Maiwall R, Harsha HC, et al. Dysregulated iron homeostasis is strongly associated with multiorgan failure and early mortality in acute-onchronic liver failure. Hepatology 2015;61(4):1306-20. 